\title{
Hematopoese extramedular: achados em tomografia computadorizada do tórax de 6 pacientes*
}

\author{
Extramedullary hematopoiesis: findings on computed \\ tomography scans of the chest in 6 patients
}

\author{
Edson Marchiori ${ }^{1}$, Dante Luiz Escuissato ${ }^{2}$, Klaus Loureiro lrion ${ }^{3}$, Gláucia Zanetti ${ }^{4}$, \\ Rosana Souza Rodrigues ${ }^{5}$, Gustavo de Souza Portes Meirelles ${ }^{6}$, Bruno Hochhegger ${ }^{7}$
}

\begin{abstract}
Resumo
Objetivo: Apresentar os achados na tomografia computadorizada do tórax indicativos de hematopoese extramedular de seis pacientes. Métodos: Foram estudadas, retrospectivamente, as tomografias de seis pacientes adultos-cinco homens e uma mulher-com idade média de 36,5 anos. Os exames foram analisados por dois radiologistas, de forma independente, e as decisões finais foram obtidas por consenso. Resultados: 0 achado mais freqüente nas tomografias foi o de massas paravertebrais inferiores, de conteúdo heterogêneo (quatro pacientes). As tomografias de dois pacientes mostravam uma massa solitária parietal e pleural. Conclusões: A hematopoese extramedular apresenta aspectos na tomografia computadorizada fortemente sugestivos do diagnóstico que, quando correlacionados com a presença de doença hematológica de base, permitem, na maior parte dos casos, dispensar a comprovação histopatológica.
\end{abstract}

Descritores: Tomografia computadorizada por raios X; Hematopoese extramedular, Mediastino/fisiopatologia, Anemia falciforme.

\begin{abstract}
Objective: To present findings on computed tomography scans of the chest indicative of extramedullary hematopoiesis in six patients. Methods: We retrospectively analyzed computed tomography scans of six adult patients-five males and one female-with a mean age of 36.5 years. Two radiologists independently reviewed the scans, and a consensus was reached in discrepant cases. Results: The most common finding in the scans was lower paravertebral masses with heterogeneous content (four patients). The scans of two patients showed a solitary parietal and pleural mass. Conclusions: There are findings in computed tomography scans that are highly suggestive of extramedullary hematopoiesis, especially when those findings correlate with underlying blood diseases. Such findings, in most of the cases, allow physicians to dispense with histopathological confirmation.
\end{abstract}

Keywords: Tomography, X-ray computed; Hematopoiesis, extramedullary; Mediastinum/physiopathology; Anemia, sickle cell.

\section{Introdução}

A hematopoese extramedular (HEM) é considerada um mecanismo fisiológico compensatório, com a formação de células sanguíneas normais fora da medula óssea, que ocorre quando esta é incapaz de suprir a demanda corporal. ${ }^{(1-6)}$ Freqüentemente, está associada a hemoglobinopatias congênitas ou a desordens de substituição medular adquiridas. ${ }^{(1,2)}$ Embora qualquer órgão possa estar envolvido, o fígado e o baço são os sítios mais comuns. ${ }^{(2,5-8)} 0$ acometimento torácico é menos freqüente e geralmente se manifesta sob a forma de massas lobuladas bilaterais, paravertebrais inferiores e com densidade de partes moles. . $^{(1,2,4,5)}$

0 diagnóstico de HEM intratorácica pode ser feito por intermédio de radiologia convencional, tomografia computadorizada (TC) ou ressonância magnética (RM), quando os achados radiológicos característicos estão presentes em associação com história de anemia crônica. ${ }^{(9)} 0$ diagnóstico diferencial é feito através das massas do mediastino poste-

\footnotetext{
* Trabalho realizado na Universidade Federal Fluminense - UFF - e na Universidade Federal do Rio de Janeiro, Rio de Janeiro (RJ) Brasil.

1. Professor Titular e Chefe do Departamento de Radiologia da Universidade Federal Fluminense - UFF - Rio de Janeiro (RJ) Brasil.

2. Professor Adjunto da Disciplina de Radiologia. Universidade Federal do Paraná -UFPR - Curitiba (PR) Brasil.

3. Consultant Chest Radiologist at The Cardiothoracic Centre NHS Trust and The Royal Liverpool and Broadgreen University Hospitals, Liverpool, Inglaterra.

4. Professora da Disciplina de Clínica Médica. Faculdade de Medicina de Petrópolis, Petrópolis (RJ) Brasil.

5. Médica do Serviço de Radiodiagnóstico. Hospital Clementino Fraga Filho e Hospital Copa D’Or, Rio de Janeiro (RJ) Brasil.

6. Médico Radiologista. Universidade Federal de São Paulo - UNIFESP - São Paulo (SP) Brasil.

7. Médico Residente em Radiologia. Complexo Hospitalar Santa Casa de Porto Alegre, Porto Alegre (RS) Brasil.

Endereço para correspondência: Edson Marchiori. Rua Thomaz Cameron, 438, Valparaíso, CEP 25685-120, Petrópolis, RJ, Brasil.

Tel 5521 2629-9076. E-mail: edmarchiori@gmail.com

Apoio financeiro: Nenhum.

Recebido para publicação em 29/11/2007. Aprovado, após revisão, em 11/2/2008.
} 
rior. Como a biópsia por agulha deve ser evitada, em função da alta vascularização deste tecido, estes métodos ganham maior importância para o diagnóstico. 0 objetivo deste trabalho foi apresentar os achados tomográficos observados em seis pacientes com HEM torácica.

\section{Métodos}

Neste estudo foram analisadas, retrospectivamente, as TC do tórax de seis pacientes com o diagnóstico de HEM intratorácica, provenientes de cinco instituições diferentes, localizadas em quatro estados da federação (Rio de Janeiro, São Paulo, Rio Grande do Sul e Paraná). Destes pacientes, cinco eram do sexo masculino e uma do feminino. A idade variou entre 19 e 59 anos, com média de 36,5 anos.

As doenças de base encontradas foram anemia falciforme (três pacientes), talassemia (dois pacientes) e esferocitose (um paciente). Dos seis pacientes, cinco eram assintomáticos do ponto de vista respiratório e um apresentava tosse e dor torácica.

0 diagnóstico foi baseado na associação dos quadros clínico e laboratorial (diagnóstico da doença de base) e nos padrões observados nas radiografias e TR. Contudo, três pacientes com quadro tomográfico menos típico realizaram biópsias da massa e tiveram a confirmação histopatológica.

As TC do tórax foram realizadas em diversos tomógrafos, com cortes axiais de 5 a $10 \mathrm{~mm}$ de espessura, com $10 \mathrm{~mm}$ de incremento, feitos em inspiração profunda, desde os ápices até as bases pulmonares. Os exames foram registrados em janela de parênquima, com largura entre 1000 e 1500 unidades Hounsfield (UH) e centro entre -500 e -750 UH. Foram registrados também em janela para o mediastino, com largura entre $300 \mathrm{e}$ $400 \mathrm{UH}$ e centro entre 40 e $60 \mathrm{UH}$.

A análise da TC foi feita por dois observadores, de forma independente, e os casos discordantes foram resolvidos por consenso. 0 estudo incluiu a avaliação das massas quanto à sua localização, distribuição e densidade, assim como à presença de outras alterações associadas.

\section{Resultados}

Dos seis pacientes, quatro apresentaram massas paravertebrais inferiores. Em três, elas eram bilaterais e relativamente simétricas, e em um era unilateral, à direita. Quanto ao conteúdo das massas, em três pacientes havia presença de tecido adiposo, sendo que em um desses o material gorduroso era bastante significativo. Em um paciente, as massas eram homogêneas, com densidade de partes moles (Figuras 1a e b, 2a e b).

Os outros dois pacientes mostravam massa parietal e pleural, ambas à esquerda, sem evidência de comprometimento paravertebral. Em um desses, a massa era homogênea (Figuras 3a e b); no outro, havia reação óssea associada.
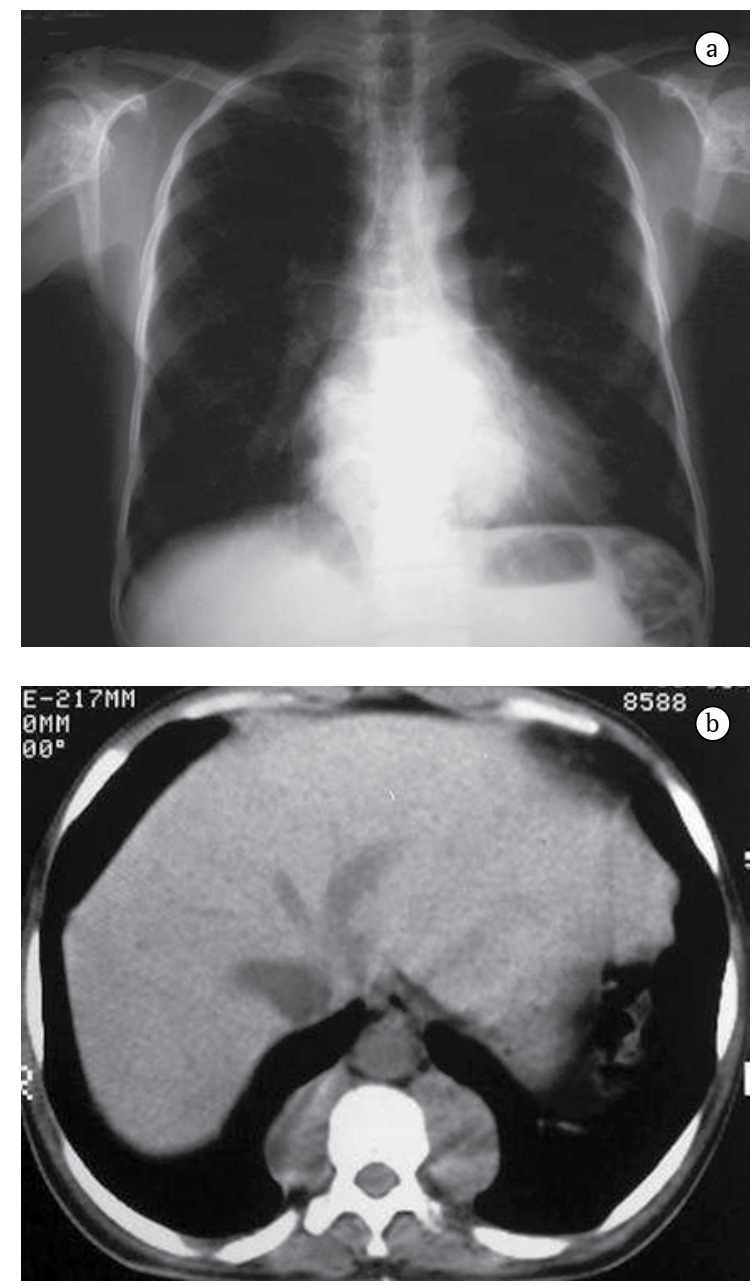

Figura 1 - a) Paciente masculino, 40 anos, com anemia falciforme. Radiografia do tórax em incidência pósteroanterior, mostrando opacidades lobuladas, com limites parcialmente bem definidos, localizadas em topografia retrocardíaca; e b) Paciente masculino, 40 anos, com anemia falciforme. Tomografia computadorizada com janela para mediastino, evidenciando que as massas estão localizadas nas regiões paravertebrais, bilateralmente. 

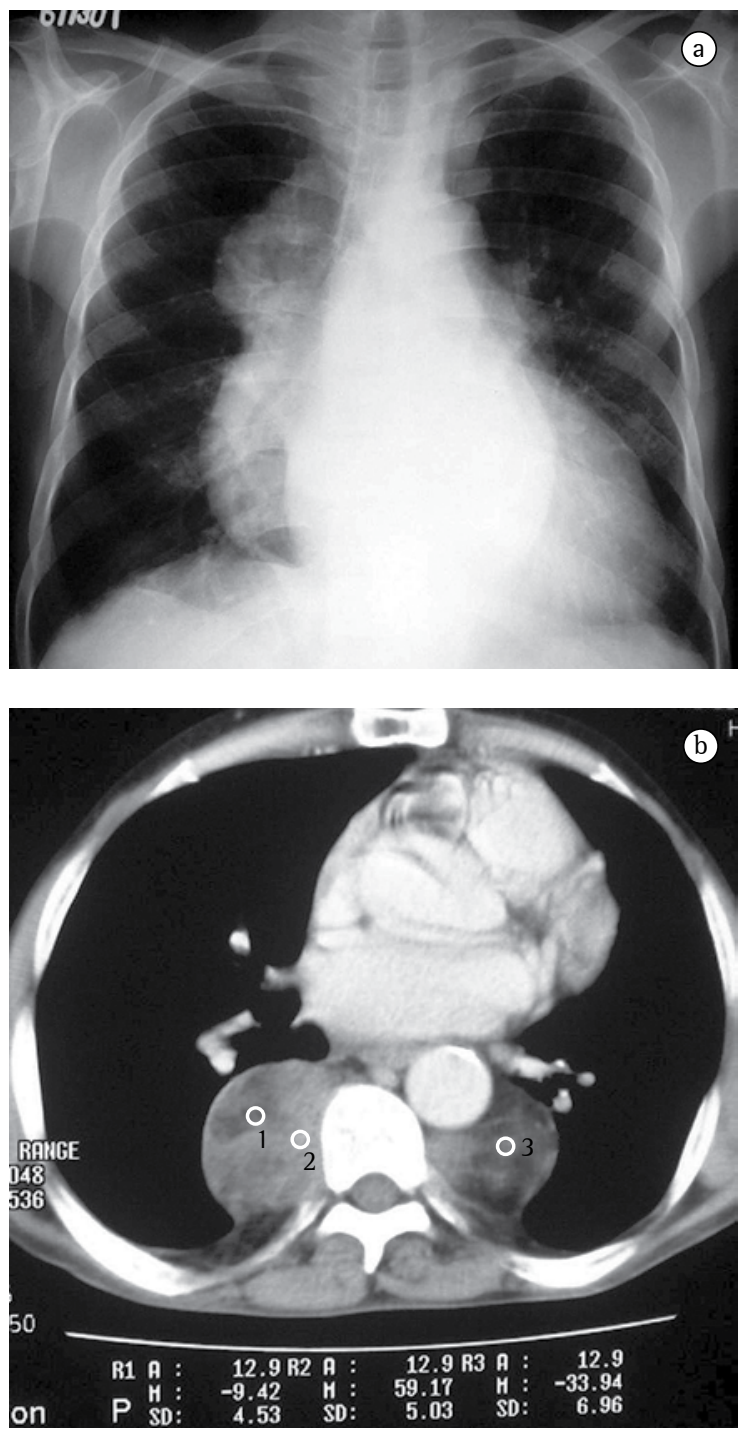

Figura 2 - a) Paciente masculino, 59 anos, com esferocitose. Radiografia do tórax em incidência posteroanterior, demonstrando volumosas massas retrocardíacas, lobuladas; e b) Paciente masculino, 59 anos, com esferocitose. Tomografia com janela para mediastino, evidenciando a heterogeneidade das massas, que apresentam áreas alternadas com densidade de partes moles e de gordura (densidades negativas).

Como achados associados, dois pacientes mostravam calcificações no baço (Figura 4). Ambos eram portadores de anemia falciforme. Dos seis pacientes, dois haviam sido esplenectomizados, e em dois a imagem do baço era normal. Alterações ósseas relacionadas à doença de base foram identificadas em três pacientes.

\section{Discussão}

AHEM está freqüentemente associada a hemoglobinopatias, como a talassemia, a anemia falciforme e a esferocitose, ou a doenças mieloproliferativas, como a leucemia, o linfoma, a mielodisplasia e a mielofibrose. ${ }^{(1,2,8,10,11)}$ A HEM é formada por áreas microscópicas difusas de tecido hematopoiético, em geral encontradas no fígado, baço e linfonodos. ${ }^{(2,5,6)}$ Outros sítios menos freqüentes são rins, pleura, pele, ovários, intestino, escleras, sistema nervoso central, espaço epidural e supra-renais. ${ }^{(2,11,12)}$ $\mathrm{Na}$ nossa casuística, três pacientes eram portadores de anemia falciforme, dois de talassemia, e um de esferocitose.

A patogênese da HEM intratorácica inclui a extrusão das células tronco da medula óssea, através do córtex fino dos corpos vertebrais e costelas, para a região subperiosteal, auxiliadas pela pressão negativa. Ocorre a proliferação destas células, não só na região da extrusão, como em outras áreas à distância, provavelmente pela proliferação de tecido hematopoiético embolizado de outras áreas, como o baço. ${ }^{(4,11)}$

0 comprometimento do tórax na HEM é pouco freqüente $\mathrm{e}^{(1,5)}$; quando ocorre, geralmente é sob a forma de massas arredondadas, lobuladas, com densidade de partes moles no mediastino posterior, sendo mais freqüente nas regiões paravertebrais inferiores. ${ }^{(1,2,4,5,9)}$ Eventualmente podem aparecer no mediastino anterior ou na pleura. ${ }^{(4)}$ Envolvimento pleural com derrame já foi relatado. ${ }^{(2,13)}$ Dos nossos pacientes, quatro apresentaram massas paravertebrais inferiores, e 2 apresentaram massas parietais e pleurais.

$\mathrm{Na}$ maioria das vezes a presença das massas não traz sintomas para o paciente. Estes podem surgir quando há compressão da medula devido à ocupação do espaço epidural, na presença de hemotórax espontâneo, ou secundário a envolvimento intersticial, quando o paciente pode desenvolver insuficiência respiratória aguda fatal. ${ }^{(3,4,10,13,14)}$ Entre nossos pacientes, cinco eram assintomáticos e um apresentou tosse e dor torácica.

Radiograficamente, a HEM intratorácica geralmente se apresenta sob a forma de múltiplas massas paravertebrais, com densidade de partes moles, na maioria das vezes bilaterais, lobuladas, com limites bem definidos e sem calcificações ou erosões ósseas associadas. ${ }^{(1,4,5,9)}$ Isso é importante para o diagnós- 

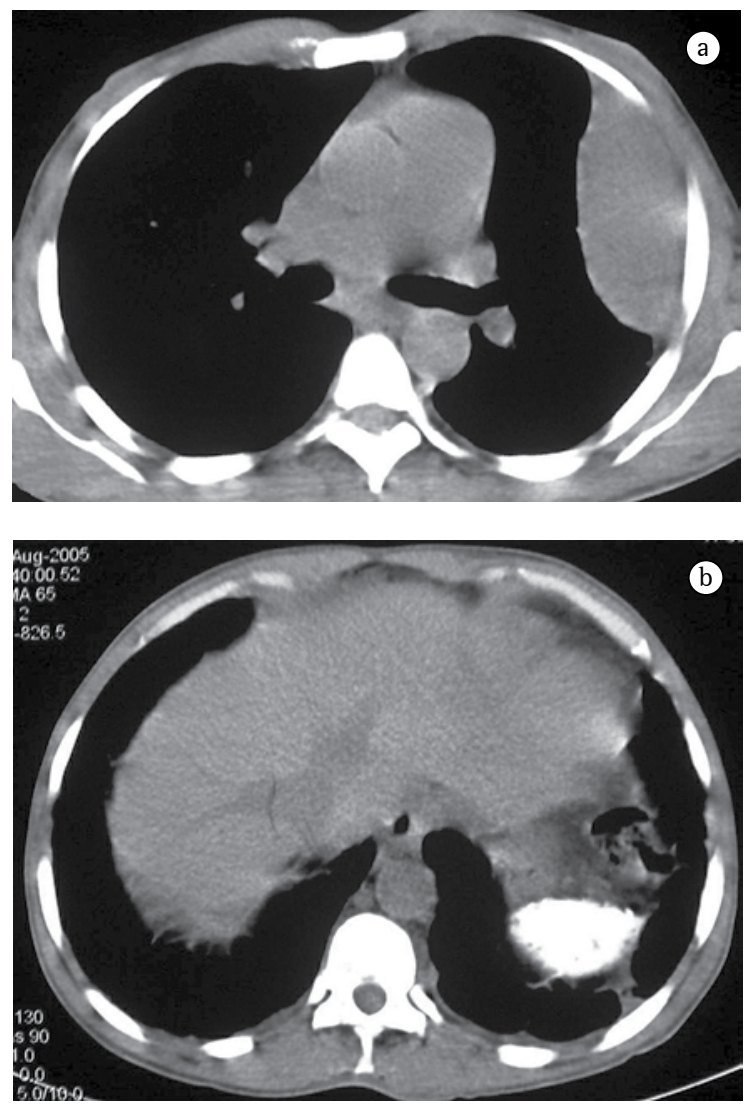

Figura 3 - a) Paciente masculino, 31 anos, com anemia falciforme, apresentando massa parietal/pleural à esquerda, homogênea; e b) Paciente masculino, 31 anos, com anemia falciforme. Corte na região abdominal superior, mostrando calcificação esplênica (autoesplenectomia).

tico diferencial de neurofibromas paravertebrais, que em geral têm alterações ósseas associadas. ${ }^{(4,10)}$ Contudo, evidências de lesões ósseas relacionadas à doença de base são achados comuns. Essas massas geralmente determinam, nas radiografias do tórax, um aspecto de duplo contorno cardíaco, lobulado, na incidência em frontal, assim como um aspecto de massa lobulada projetada sobre os corpos vertebrais inferiores na incidência em perfil. ${ }^{(13)}$

Através da TC, podem ser observadas massas com densidade de partes moles, em sua maioria homogêneas, com características semelhantes às descritas na radiologia convencional, ${ }^{(1,2,5,5)}$ que podem ou não ser realçadas após a administração do meio de contraste. ${ }^{(4,10)}$ Este método de imagem é importante para analisar a estrutura interna das lesões, particularmente quando ricas em gordura, ${ }^{(5)}$

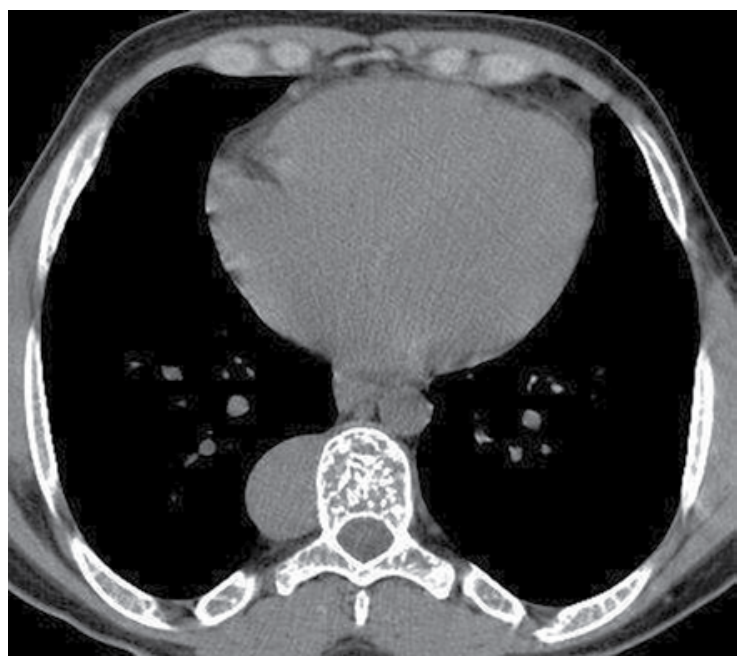

Figura 4 - Paciente masculino, 34 anos, com talassemia. Corte com janela para mediastino, onde se observa pequena massa paravertebral à direita. Observar também alterações na estrutura óssea secundárias à doença de base.

para avaliar a presença de outras massas paracostais e para detectar alterações ósseas associadas a certas doenças hematológicas, como a talassemia e a anemia falciforme. ${ }^{(6)}$ Nos casos relacionados a talassemia, anemia falciforme ou metaplasia mielóide, pode ocorrer um alargamento das cavidades medulares das costelas. ${ }^{(5)}$ Todos esses aspectos foram identificados em nossos casos. É interessante ressaltar que os achados em dois pacientes mostravam calcificações no baço, decorrentes de infartos múltiplos, com autoesplenectomia. Ambos eram portadores de anemia falciforme. Esse achado pode também auxiliar no diagnóstico diferencial.

As lesões ativas e recentes têm rica vascularização, enquanto que as antigas e inativas têm mais tecido gorduroso e deposição de ferro. Entre os dois extremos, existem várias combinações de achados, refletindo os diferentes estágios de evolução do foco hematopoiético. Os achados na TC e na RM de alguma forma traduzem essas diferenças histológicas. Lesões recentes e ativas têm densidade de partes moles na TC e intensidade de sinal intermediária na RM, tanto em $\mathrm{T} 1$ como em T2. Após a administração do meio de contraste, geralmente ocorre algum grau de impregnação nas lesões ativas. Lesões antigas e inativas podem mostrar aumento de densidade na TC, por impregnação pelo ferro, ou densidades negativas, pela infiltração gordurosa. 
$\mathrm{Na}$ RM, essas lesões podem mostrar alta intensidade de sinal tanto em T1 como em T2, pela infiltração gordurosa, ou baixa intensidade de sinal em ambas as ponderações, pela deposição de ferro. ${ }^{(4,8,12)}$

Em um estudo ${ }^{(6)}$ acompanhou-se, tomograficamente, a transformação da densidade das massas paravertebrais de um paciente em seguimento por 5 anos. Inicialmente, as massas apresentavam densidade de partes moles e, posteriormente, as massas sofreram transformação gordurosa após a esplenectomia. Essa transformação foiatribuída à resolução da desordem hemolítica e ao desaparecimento do estímulo que favorece a formação de tecido hematopoiético.

Um outro aspecto interessante descrito foi o acometimento difuso do parênquima pulmonar (traduzido tomograficamente por infiltrado em vidro fosco) e oclusão de pequenos vasos pulmonares. $\mathrm{Na}$ necropsia deste paciente foram encontrados elementos hematopoiéticos imaturos infiltrando o interstício pulmonar (achado compatível com HEM envolvendo as paredes alveolares), além de agregados de células hematopoiéticas e trombos plaquetários ocluindo os pequenos vasos pulmonares, provavelmente provenientes de outras vísceras, como o baço. ${ }^{(2)}$

0 diagnóstico de HEM intratorácica pode ser feito de forma não invasiva, com segurança, quando os achados radiológicos estão presentes e se associam à anemia crônica. ${ }^{(5)} \mathrm{A}$ biópsia por agulha deve ser evitada, pois a hipervascularização poder acarretar complicações hemorrágicas. ${ }^{(4,14)} \mathrm{Na}$ histopatologia, encontra-se geralmente tecido hematopoiético bem formado, com processo de maturação normal das três linhagens celulares. ${ }^{(14)}$

0 diagnóstico diferencial deve ser feito através das massas do mediastino posterior, incluindo tumores neurogênicos, linfoma, abscesso paravertebral, cistos extrapleurais, meningocele lateral, hiperplasia dos linfonodos mediastinais e neoplasias malignas primárias e metastáticas. ${ }^{(5,9)}$

0 tratamento é o da doença de base. ${ }^{(5)} 0$ tecido hematopoiético não deve ser removido, exceto no caso de haver compressão medular, já que ele é um mecanismo compensatório para a manutenção de uma formação eritrocitária suficiente à demanda corporal. ${ }^{(4)}$ Em casos mais extensos, a radioterapia pode ser indicada. ${ }^{(10,13)}$ A compressão medular precisa ser tratada com urgência. Derrames pleurais ou hemotórax que persistem apesar da drenagem em geral respondem bem à radioterapia torácica. ${ }^{(13)}$

Em conclusão, o achado de massas paravertebrais bilaterais bem definidas em pacientes com doença hematológica previamente diagnosticada deve alertar para a possibilidade diagnóstica de HEM.

\section{Referências}

1. Yamato M, Fuhrman CR. Computed tomography of fatty replacement in extramedullary hematopoiesis. J Comput Assist Tomogr. 1987;11(3):541-2.

2. Wyatt SH, Fishman EK. Diffuse pulmonary extramedullary hematopoiesis in a patient with myelofibrosis: CT findings. $\mathrm{J}$ Comput Assist Tomogr. 1994;18(5):815-7.

3. Chiam QL, Lau KK. Extramedullary haematopoiesis in thalassaemia major causing spinal cord compression. Australas Radiol. 2007;51(2):168-71.

4. Pornsuriyasak P, Suwatanapongched T, Wangsuppasawad $\mathrm{N}$, Ngodngamthaweesuk M, Angchaisuksiri P. Massive hemothorax in a beta-thalassemic patient due to spontaneous rupture of extramedullary hematopoietic masses: diagnosis and successful treatment. Respir Care. 2006;51(3):272-6.

5. Fielding JR, Owens M, Naimark A. Intrathoracic extramedullary hematopoiesis secondary to B12 and folate deficiency: CT appearance. J Comput Assist Tomogr. 1991;15(2):308-10.

6. Martin J, Palacio A, Petit J, Martin C. Fatty transformation of thoracic extramedullary hematopoiesis following splenectomy: CT features. J Comput Assist Tomogr. 1990;14(3):477-8.

7. Salanitri GC, Hennessy OF. Osteopetrosis with intrathoracic extramedullary hematopoiesis. AJR Am J Roentgenol. 2005;184(2):702-3.

8. Ejindu VC, Hine AL, Mashayekhi M, Shorvon PJ, Misra RR. Musculoskeletal manifestations of sickle cell disease. Radiographics. 2007;27(4):1005-21.

9. Moreira, LB, Melo AS, Marchiori E. Hematopoiese extramedular intra-torácica. Radiol Bras. 2001;34(3):177-180.

10. Lall C, Payne DK. A patient with anemia and a paraspinal chest mass. Chest. 2003;124(2):732-4.

11. Kwak HS, Lee JM. CT findings of extramedullary hematopoiesis in the thorax, liver and kidneys, in a patient with idiopathic myelofibrosis. J Korean Med Sci. 2000;15(4):460-2.

12. Tsitouridis J, Stamos S, Hassapopoulou E, Tsitouridis K, Nikolopoulos P. Extramedullary paraspinal hematopoiesis in thalassemia: CT and MRI evaluation. Eur J Radiol. 1999;30(1):33-8.

13. Bowen EF, Marsh JC, Sandu C, Young M, Rayner CF. An elderly female with dyspnoea and anaemia. Eur Respir J. 2000;16(3):566-9.

14. Chu KA, Lai RS, lee $\mathrm{CH}$, Lu JY, Chang HC, Chiang HT. Intrathoracic extramedullary haematopoiesis complicated by massive haemothorax in alpha-thalassaemia. Thorax. 1999;54(5):466-8. 